\title{
User Privacy in RFID Networks
}

\author{
Dave Singelée ${ }^{1}$, Stefaan Seys ${ }^{2}$ \\ ${ }^{1}$ ESAT - SCD - COSIC, Katholieke Universiteit Leuven - IBBT \\ Kasteelpark Arenberg 10, 3001 Heverlee-Leuven, Belgium \\ Dave.Singelee@esat.kuleuven.be \\ ${ }^{2}$ PricewaterhouseCoopers Enterprise Advisory \\ Woluwedal 18, 1932 Sint-Stevens-Woluwe, Belgium \\ Stefaan.Seys@pwc.be
}

\begin{abstract}
Wireless RFID networks are getting deployed at a rapid pace and have already entered the public space on a massive scale: public transport cards, the biometric passport, office ID tokens, customer loyalty cards, etc. Although RFID technology offers interesting services to customers and retailers, it could also endanger the privacy of the end-users. The lack of protection mechanisms being deployed could potentially result in a privacy leakage of personal data. Furthermore, there is the emerging threat of location privacy. In this paper, we will show some practical attack scenarios and illustrates some of them with cases that have received press coverage. We will present the main challenges of enhancing privacy in RFID networks and evaluate some solutions proposed in literature. The main advantages and shortcomings will be briefly discussed. Finally, we will give an overview of some academic and industrial research initiatives on RFID privacy.
\end{abstract}

\section{Introduction}

RFID (Radio Frequency Identification) is used to denominate a digital identifier that can be read using RF signals. There are three main types: passive RFID tags that require an external power source to operate, active tags that have an on-board battery and battery assisted passive (BAP) that require an external source to wake-up, but have a larger forward link capacity.

Today, RFID technology is used in a multitude of applications, for example:

- $\quad$ IT Asset Tracking

- RFID passports

- Mobile Credit Card payment systems

- Transportation payment systems

- Barcode replacements: product tracking, transportation and logistics

- Timing and tracing at sporting events (lap timing)

- Animal identification

As most RFID applications require massive deployment of tags, the cost of tags should be minimized. Because of this, security features have initially been close to non-existent and none of the basic security features one expects from a wireless communication system (such as au- 
thentication and encryption) were part of the first designs. As this opens the door for a large number of attacks and privacy issues, researchers have started to investigate possible solutions. The main challenges these researchers have to tackle are explained in Section 2.

After a short selection of real-world security issues, we will present possible privacy enhancing techniques for RFID systems.

\subsection{Selection of real-world security issues of RFID sys- tems}

Privacy concern related to the large scale deployment of RFID tags in consumer products

Replacing the ubiquitous bar code with RFID tags has many advantages, both for suppliers, retailers and consumers. Stock management and shipping can be further automated and your washing machine will detect whether you have combined coloured clothes and whites or not. Nevertheless, privacy concerns have been raised since day one. In general, bar codes cannot be read remotely, while this is exactly the main advantage of RFID tags. This means that the consumer will be carrying around a "cloud" of RFID tags at anytime that will leak information:

- The "cloud" itself will act as a unique identifier and can be used to track this individual;

- Certain RFID tags might reveal that you carry expensive items in your purse;

- Other RFID tags might reveal personal data such as medication you are taking, etc.

These concerns are not ignored by the legislation. For example, the European Commission recently issued "RFID Privacy Recommendations". One of the recommendations of the EC is that consumers should be actively involved when the RFID tag information is used. How this translates in technology still remains to be seen.

\section{Cloning of the RFID chip in the UK's biometric passport}

In 2006, Lukas Grunwald demonstrated at the Black Hat conference how to clone a UK biometric passport. After studying the publicly available standard used for the UK ePassports, he was able to read the data stored in the RFID of a genuine UK passport and copy it into a blank RFID chip. In this case, the complete RFID chip was cloned and the clone can no longer be distinguished from the original. Note that this attack could potentially be used to clone passports without having physical access to the passport.

\section{Technological Challenges}

RFID security is particularly difficult to design because of the specific properties of RFID chips and systems.

Virtually all applications of RFID systems require that the RFID chips are cheap to manufacture. Because of this, the resources available on typical RFIC chips are ultra limited. This is true on all levels: ultra low-power (in the best case a tiny battery), tiny CPU with limited processing power, very limited memory, very small bandwidth, no internal clock, etc. This has a large influence on the use of cryptographic primitives that can be deployed. In general, public key cryptography cannot be used in RFID networks, as these techniques are too computationally demanding.

Another consequence of the cheap production process is that RFID chips will not achieve the same level of physical security properties as their more expensive smartcard cousins. Because of this, they are not tamper resistant and thus more susceptible to physical attacks. 
Another property of RFID systems is that are carried around by consumers in public places. This means that attackers will have ample opportunity to execute their attack. Furthermore, by employing tailored tag readers, attackers are able to eavesdrop on an RFID response from a much larger distance than the typical user expects. More advanced attacker models include active attackers (that can modify or replay communications) or even man-in-the-middle attackers that spoof a response to a tag reader over a large-distance relay using other communication means (e.g., forwarding the reader request over a cell phone connection to a tag at a completely different location and relaying the answer the same way back).

For the time being, there is no actual RIFD communication infrastructure that allows the use of central servers to assist with security (e.g., key servers to assist in key management).

All these limitations complicate the design of privacy preserving solutions for RFID systems.

\section{Privacy Enhancing Solutions for RFID Networks}

Various solutions to enhance the privacy of RFID applications have been proposed in the literature, taking into account the constraints on cost, energy efficiency and computational power. We will now give an overview of some of the proposed solutions, and evaluate their main advantages and drawbacks.

\subsection{RFID Kill Command}

One of the most straightforward solutions to provide privacy to the end-users of RFID tags, is just to permanently disable the tag's ability to transmit information (i.e. kill the tag) when it should not be used anymore (e.g., when the product with the RFID tag attached to it, leaves the store). This kill function is typically performed on a software basis (by sending a kill command to the tag). To protect against accidental or malicious killing of RFID tags, the kill function is password protected. To destroy the tag permanently, the kill command has to be accompanied by a tag-specific password, hard coded in the RFID tag. In the EPCglobal standard [EPC08], the kill password is 32 bits in length. Once this command is sent, there are three scenarios:

1. The password is correct and the tag is killed successfully,

2. The password is incorrect, and the tag ignores the kill command, or

3. The password is correct, but the tag did not gain enough power to complete the kill function. The tag then sends an error message back to the reader.

The main technical challenge associated with kill commands is that they imply a vulnerability if kill-passwords are not properly secured. This could result in an attacker deactivating the RFID tag functionality and hence threaten the supply chain transactions [SpEv09].

The main advantage of using the RFID kill command is that it is a very straightforward solution and offers the best privacy guarantees for the end-user. From the moment it has been disabled, it cannot be tracked anymore. There are however also some drawbacks. The kill command solution is useless for situations such as access control, where legitimate readers need to verify the authenticity of the tag. This solution disallows transactions beyond the point of sale and hence conflicts with all use cases propagated by industry for after-sales services. 


\subsection{Physical Privacy}

An alternative to sending a kill command to the RFID tag, is physically destroying the tag (i.e. a hardware kill function) when it should not be used anymore. IBM has proposed to use scratch-off material (similar to that found on lottery tickets) for the antenna of the RFID tag, so that customers can visually check that the tag's ability to transmit information has been disabled [IBM06, KaMo05].

The solution proposed by IBM is an example of physical privacy. The main idea of this solution is to use the physical RFID tag structures to permit a consumer to disable a tag by mechanically altering the tag in such a way that the ability of a reader to interrogate the RFID tag by wireless mean is inhibited [KaMo05]. These disabled tags are also called "clipped tags". An additional and desirable feature is to be able to reactivate the tag by using a physical contact channel. Such a reactivation requires deliberate actions of the RFID owner. Reactivation of the RFID tag should not be possible without the owner's knowledge and permission, unless the tag is stolen.

A similar approach to the "clipped tags" is shielding the wireless transmission of a RFID tag. By attaching a piece of metal to the RFID tag, one can construct a Faraday cage that blocks all wireless communication, and hence makes the tag invisible to readers. By removing the metal, the tag can be detected again. This is a simple solution to provide configurable privacy. However the main drawback is that such a Faraday cage is impractical if the RFID tags are attached to large objects (e.g., a pair of trousers).

The main advantage of the physical privacy solution is that it offers a visual confirmation to the customers that the tag is privacy protected. Mechanisms that enable the reactivation of a tag have the additional benefit that they support the industrial use cases for after-sales services. Physical privacy hence seems to be an interesting solution to enhance RFID privacy. There are however some drawbacks, including the extra production cost (to make tags that can be clipped) and the need of complex (technical) user interactions to reactive the tag. There is also the risk of sabotage, where a user disables the tag before the point of sale. In some scenarios, this could be beneficial to the customer (e.g., in a supermarket, where RFID tags could be used to scan all purchased products).

\subsection{Cryptographic Authentication Protocol}

Instead of physically altering the RFID tag or sending software commands to kill it, one can also deploy cryptographic techniques to solve the problem. There are two goals one typically wants to achieve by deploying a cryptographic authentication protocol in RFID networks: security (to guarantee the authenticity of tags being accepted by the authentication protocol) and privacy.

The goal of a privacy-preserving cryptographic authentication protocol is to enable a RFID tag to prove its identity to a legitimate reader without revealing its unique identity to any third party. Such a third party can be passive (eavesdropping the communication), but can also perform more active attacks (such as modifying or replaying the communication), or even perform man-in-the-middle attack. Designing an authentication protocol that tackles all these attacks is a challenging task.

There are essentially two approaches that have been proposed to solve this problem [SaVW09]. A first approach is to perform a challenge-response protocol between the RFID reader and the tag. This solution is schematically depicted in Fig.1. The protocol starts with the 
reader sending a challenge to the authenticating tag. The latter then computes a response, dependent on the challenge, by using a shared secret key (shared between the tag and the reader). In the last phase of the protocol, the reader performs an exhaustive search on all the keys its shares with RFID tags. If a match is found, it knows which particular RFID tag performed the authentication protocol. If none of the keys match, the authentication protocol fails. Instead of conducting a single round of a challenge-response protocol with both the challenge and the response having a bit length of $n$ bits, one can also conduct $n$ rounds using single bit challenges and responses. Examples of RFID challenge-response protocols are [EnHJ04, MoWa04, Tsud06, WSRE03].

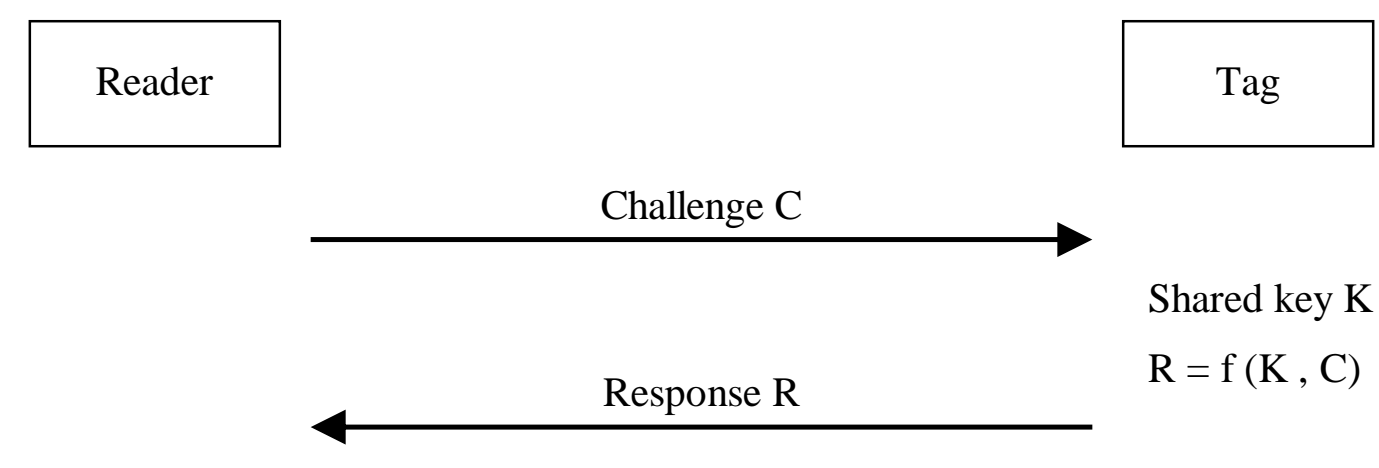

For all keys $\mathrm{K}_{\mathrm{i}}$ :

$\mathrm{f}\left(\mathrm{K}_{\mathrm{i}}, \mathrm{C}\right)=\mathrm{R} ?$ ?

Fig. 1: RFID challenge-response authentication protocol

A second approach that has been proposed in the literature, is to have the tag use a temporary identifier (also called a "pseudonym") to identify itself. In each separate run of the authentication protocol, the pseudonym is updated (typically by using a secret key shared between tag and reader). This update is done in such a way that the different pseudonyms used by the tag, are unlinkable from the point of view of a third party. Only the legitimate readers can link the pseudonyms of a tag to each other, and hence identify the tags. This approach requires synchronization between a tag and all the readers in the system (otherwise, the reader will expect another identifier than the current pseudonym used by the tag). Various pseudonym schemes for RFID have been proposed in literature (see e.g., [Dimi05, HeMu04, JuWe05, MoSW05, SoMi08]).

The main advantage of using a cryptographic authentication protocol in RFID networks is that privacy is preserved automatically. Neither the end-user nor the retailer does have to perform any additional tasks. An additional benefit is that the RFID tag does not have to be disabled or altered. The tag continues to transmit (anonymous) data, even after the store exit, and privacy is always on. This solution hence supports the industrial use cases for after-sales services. There are however also some disadvantages of using cryptographic authentication protocols in RFID networks. The efficiency of using a challenge-response protocol with a shared symmetric key depends on the total number of tags in the system. A large number of tags will be inefficient and not scalable. The protocols that use temporary pseudonyms that are updated in each run of the protocol, are vulnerable to denial-of-service attacks that desynchronize the tag and the reader [JuWe06]. Another drawback is that several authentication protocols have been demonstrated to be broken and are hence not privacy preserving (e.g., see [Avoi05, FrSh09, JuWe07, Vaud07] for a security analysis of various RFID authentication protocols). 


\subsection{Distance Bounding Protocols}

One of the attacks that need to be prevented when conducting a RFID authentication protocol, is a man-in-the-middle attack. By performing such an attack, the adversary can impersonate a legitimate tag to a RFID reader, even when both devices are not in each other's proximity. To prevent man-in-the-middle attacks, several researchers have proposed to incorporate the distance between RFID tag and reader in the authentication protocol. This leads to the concept of distance bounding protocols. These protocols enable a verifying party to determine an upper bound on the distance between itself and a prover, who claims to be within a certain range. By combining cryptographic and physical properties, one can design a secure authentication protocol that is resistant to man-in-the-middle attacks.

Secure distance bounding protocols typically measure the time of flight to determine an upper bound on the distance between prover and verifier. This measurement is performed during a challenge-response protocol, the main building block of the distance bounding protocol. This challenge-response protocol is executed $n$ times. During each of the $n$ rounds, the time between sending a challenge and receiving the response is measured. Multiplying the time of flight with the propagation speed of the communication medium gives the distance between prover and verifier. Several secure distance bounding protocols have been proposed in the literature (e.g., see [BrCh93, HaKu05, SiPr07]).

Since distance bounding protocols are a specific type of authentication protocols, they have the same properties as the latter (see Section 3.3 for more details). An additional advantage are their interesting security properties, as a consequence of implicitly incorporating the location between both devices. The major drawback is the large implementation costs. Distance bounding protocols impose very strict requirements on the hardware. It is unclear if these requirements can be achieved with the current state-of-the-art RFID technology.

\subsection{Privacy Agents}

Several researchers have introduced the concept of privacy agents. The idea is that consumers carry their own privacy-enforcing devices for RFID instead of relying on public RFID readers to enforce privacy protection. Users delegate the privacy management to a privacy agent that mediates tag-reader communication based on a privacy policy [SpEv09]. Three approaches have been proposed to conduct privacy agent schemes: using a RFID proxy, using a blocker tag, and using anonymizers.

\subsubsection{RFID Proxy}

The basic idea is that users carry a RFID proxy (also called "watchdog" or "RFID guardian") that acts as a personal RFID firewall [JuSB05, RiCT05]. Users delegate the privacy management to the RFID proxy, which implements their privacy policy. These policies are typically defined as follows: "Only reader $x$ and $y$ may scan tag $t$ at location $z$ ".

All requests from the RFID reader to the tag are intercepted by the RFID proxy, which then analyzes the request and performs the necessary actions based on the user's privacy policy. If access is granted, the request can be forwarded to the tag, or the RFID proxy can reply itself. In other words, the RFID proxy then simulates the tags that are under its control. If a reader is not authorized to access a person's tags, then the RFID proxy selectively jams the wireless communication between the reader and the tag.

The main challenges of this approach are: 
- techniques to securely have the RFID proxy acquire or release control over the tag,

- guarantee the correctness of authentication claims performed by the proxy,

- effectively cut off the tag-reader communication when access to a tag is denied, and

- having users specify their privacy policy and import this policy correctly into the RFID proxy.

Further research is necessary to tackle these challenges efficiently.

\subsubsection{Blocker Tag}

A simple but effective privacy policy could be to have two privacy states: on or off. When privacy is turned on, the tag is undetectable by the reader. When privacy is turned off, the reader can query the tag. This privacy policy can be implemented by a blocker tag. The concept was proposed by Juels, Rivest, and Szydlo to preserve privacy in RFID networks [JuRS03]. The basic principle of their solution is that a tag can act in two modes: the normal mode where it emits its unique identifier (e.g., in the shop), and a privacy mode (e.g., beyond the point of sale). To enable a tag to be in privacy mode, a blocker tag is used. When activated, it prevents undesirable scanning of RFID readers.

This is done by exploiting the anti-collision protocol used in RFID communication. To ensure that tag signals do not interfere with one another during the scanning process, the reader first ascertains what tags are present, and then addresses tags individually. A blocker tag impedes RFID scanning by simulating collisions. So in other words, the blocker tag just simulates that all possible RFID tags are present. It can also block a subset of all possible tags (e.g., only the tags which have a unique identifier starting with a ' 1 '). The blocker tag does not engage in an active form of jamming. Rather, by participating in the tag-reading process in a non-compliant way, it performs what may be thought of as a kind of passive jamming. Additional technical details on this technique can be found in [JuRS03].

A blocker tag creates a physical region of privacy protection in which a reader is incapable of reading RFID tags. It can however also be used as a malicious tool, to perform denial-ofservice attacks. By employing blocker tags, the attacker can shield all tags (or a particular subset) from being read by a reader. This limits the use in a commercial environment.

\subsubsection{Anonymizers}

Another approach of delegating privacy management to a privacy-enforcing device, is the concept of anonymizers. The basic idea is to use temporary pseudonyms instead of the unique identifier of the tag in the tag-reader communication. The difference with employing cryptographic authentication protocols (as has been discussed in Section 3.3), is that the tags do not update their temporary identifier (i.e. the pseudonym) themselves because of efficiency reasons. Instead, they delegate this task to the anonymizer, a special device owned by the enduser. This device can be a dedicated device, but can also be implemented on a mobile phone or a PDA running anonymizer software [SaVW09]. Both the tag, reader and the anonymizer can then conduct an anonymizer-enabled RFID authentication protocol. Each time a tag is queried, it replies with its temporary pseudonym that it has stored in its memory. To avoid tracking, the pseudonym has to be updated regularly. This is done by the anonymizer and the tag conducting a protocol, where the anonymizer authenticates itself and sends an updated pseudonym to the tag. The tag always uses the current pseudonym to authenticate itself to the reader. By using an anonymizer, the user can implement its own privacy policy, and choose how regular the pseudonym of the tag should be updated. 
Anonymizers are and interesting solution to preserve privacy in RFID networks. Since a mobile phone (or another personal mobile device) can be used as an anonymizer, the cost is reasonable low. One of the major drawbacks are the security properties of these schemes: most anonymizer-enabled RFID authentication protocols proposed in the literature are subject to impersonation attacks [SaVW09].

\section{Academic and Industrial Research Projects}

Security and privacy in RFID networks is an important research area that is intensively studied in the academic and industrial world. This is illustrated by the large number of academic and industrial research projects on this topic. Without having the intention to be complete, we give a brief overview of some of these initiatives:

- RFID Guardian: The RFID Guardian Project is a collaborative project focused upon providing security and privacy in Radio Frequency Identification (RFID) systems. The goals of this project are to investigate the security and privacy threats faced by RFID systems, design and implement real solutions against these threats, and investigate the associated technological and legal issues. The website of the project is: http://www.rfidguardian.org/

- $\quad$ RFID Ecosystem: The RFID Ecosystem is a large-scale project with participants from various research groups at the University of Washington's Department of Computer Science and Engineering. The project investigates user-centered RFID systems in connection with technology, business, and society. Past research on user applications of RFID has been limited to short-term technology and user studies in restricted scenarios. In contrast, the RFID Ecosystem provides a living laboratory for long-term, in-depth research in applications, databases, privacy, security, and systems. The project website is: http://rfid.cs.washington.edu/

- PEARL: The PEARL project performs research on a privacy enhanced security architecture for RFID labels. The goal of this project is to develop tools and methodologies as well as their theoretical foundations for using RFID systems while preserving the user's privacy. In this project, researchers will formally model the relevant privacy and security properties, develop new privacy enhancing protocols for the extremely resource constrained RFID environment, shape a context in which the user can check the privacy policies enforced by the RFID-based application, and develop methods to secure the integration of RFID tags and the back office applications that will support them. The project website is: http://www.cs.ru.nl/pearl/

- BCRYPT: This project intends to perform fundamental research into a number of selected disciplines that intend to address the information security challenges. WP7 of this project focuses on the application area RFID. This work package brings together research from the following fundamental areas: cryptographic algorithms and protocols, secure software and secure hardware. In this project, researchers investigate how privacy can be provided by the cooperation of new algorithms together with an extremely efficient hardware and software implementation. The website of the project is: https://www.cosic.esat.kuleuven.be/bcrypt/

- $\quad$ STOA project RFID \& Identity Management: The STOA project "RFID \& Identity Management" aims to provide insight into how Radio Frequency Identification is experienced by European citizens, draw a future scenario, and formulate challenges for this rapidly emerging technology. The final deliverable of the project can be found on: http://www.europarl.europa.eu/stoa/publications/studies/stoa182 en.pdf 
- RFID-AP: The RFID-AP project performs research on the range of security threats to applications based on the deployment of RFID-tags and concentrates on two particular issues: those of authentication and privacy. The goal of RFID-AP is to design and prototype cryptographic algorithms and secure protocols for RFID deployment. Such algorithms and protocols could be used individually, or in combination. The project website is: http://www.rfid-ap.fr/

\section{Conclusion}

In this article, we have given an overview of privacy enhancing solutions for RFID systems. From this overview, it is clear that providing privacy within the limitations dictated by RFID technology is not straightforward and further research will be required to solve the issue. We have completed this report with an overview of ongoing research projects that try to advance the field of privacy and security for RFID systems.

\section{Acknowledgements}

Dave Singelée's work was supported by the Interdisciplinary institute for Broadband Technology (IBBT) and in part by the Concerted Research Action (GOA) Ambiorics 2005/11 of the Flemish Government and by the IAP Programme P6/26 BCRYPT of the Belgian State (Belgian Science Policy).

\section{References}

[Avoi05] Avoine Gildas: Adversary Model for Radio Frequency Identification. In: LASEC Technical Report, 2005-001, Swiss Federal Institute of Technology (EPFL). 2005, 14 pages.

[BrCh93] Brands Stefan, Chaum David: Distance-Bounding Protocols. In: Advances in Cryptology - EUROCRYPT 1993, Lecture Notes in Computer Science, LNCS 765, Springer-Verlag. 1994, p. 344-359.

[Dimi05] Dimitriou Tassos: A lightweight RFID protocol to protect against traceability and cloning attacks. In: Proceedings of the $1^{\text {st }}$ International Conference on Security and Privacy for Emerging Areas in Communications Networks, IEEE Computer Society. 2005, p. 59-66.

[EnHJ04] Engberg Stephan, Harning Morten Borup, Jensen Christian Damsgaard: Zeroknowledge Device Authentication: Privacy and Security Enhanced RFID Preserving Business Value and Consumer Convenience. In: Proceedings of the Second Annual Conference on Privacy, Security and Trust. 2004, p. 89-101.

[EPC08] EPC global: Class 1 Generation 2 UHF Air Interface Protocol Standard version 1.2.0. In: http://www.epcglobalinc.org/home. 2008, 108 pages.

[FrSh09] Frumkin Dmitry, Shamir Adi. Un-trusted-HB: Security Vulnerabilities of Trusted-HB. In: Proceedings of the $5^{\text {th }}$ Workshop on RFID Security. 2009, p. 6271.

[HaKu05] Hancke G.P., Kuhn M.G.: An RFID Distance Bounding Protocol. In: Proceedings of the $1^{\text {st }}$ International Conference on Security and Privacy for Emerging Areas in Communications Networks, IEEE Computer Society. 2005, p. 67-73. 
[HeMu04] Henrici Dirk, Müller Paul: Hash-based enhancement of location privacy for radiofrequency identification devices using varying identifiers. In: Proceedings of the $2^{\text {nd }}$ IEEE International Conference on Pervasive Computing and Communications Workshops, IEEE Computer Society. 2004, p. 149-153.

[IBM06] IBM: IBM Licenses Clipped Tag RFID Technology to Marnlen RFiD. In: http://www-03.ibm.com/press/us/en/pressrelease/20592.wss. 2006.

[JuRS03] Juels Ari, Rivest Ronald, Szydlo Michael: The blocker tag: selective blocking of RFID tags for consumer privacy. In: Proceedings of the 10th ACM Conference on Computer and Communications Security, ACM. 2003, p. 103-111.

[JuSB05] Juels Ari, Syverson Paul, Bailey Daniel: High-Power Proxies for Enhancing RFID Privacy and Utility. In: Proceedings of the 5th International Workshop on Privacy Enhancing Technologies, Lecture Notes in Computer Science, LNCS 3856, Springer-Verlag. 2005, p. 210-226.

[JuWe05] Juels Ari, Weis Stephen: Authenticating pervasive devices with human protocols. In: Advances in Cryptology - CRYPTO 2005, Lecture Notes in Computer Science, LNCS 3621, Springer-Verlag. 2005, p. 293-308.

[JuWe07] Juels Ari, Weis Stephen: Defining Strong Privacy for RFID. In: Proceedings of the $5^{\text {th }}$ IEEE International Conference on Pervasive Computing and Communications Workshops, IEEE Computer Society 2007, p. 342-347.

[KaMo05] Karjoth Günther, Moskowitz Paul: Disabling RFID tags with visible confirmation: clipped tags are silenced. In: Proceedings of the 2005 ACM workshop on Privacy in the electronic society, ACM. 2005, p. 27-30.

[MoSW05] Molnar David, Soppera Andrea, Wagner David: A Scalable, Delegatable Pseudonym Protocol Enabling Ownership Transfer of RFID Tags. In: Proceedings of the 12th International Workshop on Selected Areas in Cryptography, Lecture Notes in Computer Science, LNCS 3897, Springer-Verlag. 2005, p. 276-290.

[MoWa04] Molnar David, Wagner David: Privacy and security in library RFID: Issues, practices, and architectures. In: Proceedings of the $11^{\text {th }}$ ACM Conference on Computer and Communications Security, ACM. 2004, p. 210-219.

[RiCT05] Rieback M., Crispo B., Tanenbaum A.: RFID Guardian: A batterypowered mobile device for RFID privacy management. In: Proceedings of the $10^{\text {th }}$ Australasian Conference on Information Security and Privacy, Lecture Notes in Computer Science, LNCS 3574, Springer-Verlag. 2005, p. 184-194.

[SaVW09] Sadeghi A-R., Visconti I., Wachtsmann C.: Efficient RFID Security and Privacy with Anonymizers. In: Proceedings of the $5^{\text {th }}$ Workshop on RFID Security. 2009, p. 153-172.

[SiPr07] Singelée Dave, Preneel Bart: Distance Bounding in Noisy Environments. In: Proceedings of the 4th European Workshop on Security and Privacy in Ad Hoc and Sensor Networks, Lecture Notes in Computer Science, LNCS 4572, SpringerVerlag. 2007, p. 101-115.

[SoMi08] Song Boyeon, Mitchell Chris J.: RFID authentication protocol for low-cost tags. In: Proceedings of the $1^{\text {st }}$ ACM Conference on Wireless Network Security, ACM. 2008, p. 140-147. 
[SpEv09] Spiekermann Sarah, Evdokimov Sergei: Critical RFID Privacy-Enhancing Technologies. In: IEEE Security and Privacy, Vol. 7, no. 2, IEEE Computer Society. 2009, p. 56-62.

[Tsud06] Tsudik Gene: YA-TRAP: Yet Another Trivial RFID Authentication Protocol. In: Proceedings of the $4^{\text {th }}$ IEEE International Conference on Pervasive Computing and Communications Workshops, IEEE Computer Society. 2006, p. 640-643.

[Vaud07] Vaudenay Serge: On Privacy Models for RFID. In: Advances in Cryptology ASIACRYPT 2007, Lecture Notes in Computer Science, LNCS 4833, SpringerVerlag. 2007, p. 68-87.

[WSRE03] Weis S., Sarma S., Rivest S., Engels D.: Security and privacy aspects of low-cost radio frequency identification systems. In: Proceedings of the $1^{\text {st }}$ International Conference on Security in Pervasive Computing, Lecture Notes in Computer Science, LNCS 2802, Springer-Verlag. 2003, p. 454-469.

\section{Index}

RFID, privacy, personal data, location privacy, pseudonyms, authentication, anonymizer 